\title{
Integrating Patient-Generated Health Data in an Electronic Medical Record: Stakeholders' Perspectives
}

\author{
Katherine BLONDON ${ }^{\mathrm{a}, \mathrm{b}, 1}$ and Frederic EHRLER ${ }^{\mathrm{a}}$ \\ ${ }^{a}$ University Hospitals of Geneva, Switzerland \\ ${ }^{\mathrm{b}}$ University of Geneva, Swtizerland
}

\begin{abstract}
Patient-generated health data (PGHD), when shared with the provider, provides potential as an approach to improve quality of care. Based on interviews and a focus group with stakeholders involved in PGHD integration in the electronic medical record (EMR), we explore the benefits, barriers and possible risks. We propose solutions to address liability concerns, such as clarifying patient and provider expectations for the analyses of PGHD and emphasize considerations for future steps, which include the need to screen PGHD for patient safety.
\end{abstract}

Keywords. Patient-generated health data (PGHD), patient-reported outcomes, electronic medical record, data visualization

\section{Introduction}

Patient-generated health data (PGHD) are defined as "health-related data created, recorded, or gathered by or from patients (or family members or other caregivers) to help address a health concern" by the Office of the National Coordinator for Health Information Technology (ONC) [1]. PGHD can play an important role in understanding a patient's health when they are away from healthcare providers: it is well known that certain clinical parameters such as blood pressure measurements can vary in the presence of a healthcare provider, an effect known as the white coat effect. Adapting treatments solely based on the blood pressure measurements at a doctor's practice may lead to overtreatment, with a risk for hypotensive or other side effects. Patients can measure their own blood pressure values at home nowadays, and then share this information with their doctor to help adapt the medication. When we consider that patients spend more than $99 \%$ of their time away from healthcare providers, the value of PGHD to help adapt healthcare management may seem obvious, yet many elements hinder the use of these data in current care [2].

There are several types of PGHD reported in prior literature [3]. One type of PGHD concerns clinical parameters such as blood pressure and glucose levels that patients collect on their own: it may be self-driven, or recommended by a care-provider. It is often quantitative, with datasets that can have occasional points or a very large number of data points (i.e. glucose monitoring). Another type of PGHD are patient-reported outcomes

${ }^{1}$ Corresponding Author, Katherine Blondon, Medical Directorate, University Hospitals of Geneva 1205 Geneva, Switzerland; E-mail: Katherine.blondon@hcuge.ch. 
(PROs), which are often questionnaires that patients fill out, once or several times. They can be for screening or monitoring, used for patient care or for institutional reporting (ex: perceived pain assessments) often collected through the use of questionnaires that are filled out by patients. It is clear that PGHD can be a source of data for several different goals: PROs are commonly used by healthcare professionals to collected data for clinical studies, or to monitor cohorts. PGHD can allow patients to share and compare experiences among themselves, or as mentioned above, to improve the care that they receive. They can also help institutions improve the quality of care provided at a population level.

With the advent of mobile and connected devices, the amount of PGHD has increased tremendously [4]. Although prior studies underline the potential of PGHD in helping improve the care patients receive, very little PGHD is integrated in electronic medical records (EMRs) [5]. Healthcare professionals' use of PGHD is hindered by several factors. In this paper, we report our findings from encounters with the stakeholders involved in the integration of PGHD for patient care [6]. We propose a synthesis of the issues to overcome, and suggest solutions that need to be considered when integrating PGHD into EMRs.

\section{Methods}

We identified and encountered the stakeholders for the integration of PGHD in the EMRs, including physicians, patients, computer scientists, and a legal services representative (purposive sampling). We used a semi-structured approach with two scenarios to illustrate the different types of PGHD, to help contextualize and to drive the discussion about barriers, risks and possible solutions in integrating PGHD into a patient's care. We conducted both individual interviews and a focus group with physicians, due to the stakeholders' limited availability during the COVID crisis. We conducted a thematic analysis of our interviews and report our findings and proposed solutions [7].

The first scenario was about the use of questionnaires, which can be one-shot or repeated to detect changes over time. These questionnaires can be a clinical score with a quantitative result, or a survey with multiple choice questions or free text responses. After discussing general principles, we proposed the example of a questionnaire including questions about suicidal thoughts: a patient fills this questionnaire in after office hours, indicating distress and high risk of suicide, and even attempts suicide during the night. We collected responses to this scenario, enquiring about initial reactions, preventive measures and legal considerations.

The second scenario illustrated the use of quantitative, patient-measured parameters, such as glucose, weight or blood pressure results. These parameters typically contain a large number of data points, and can potentially require a rapid clinical response (e.g., low blood sugar result). In this scenario, we considered a patient with diabetes, who shares his glucose levels with his healthcare team. Over the course of three days, the patient presents low glucose values at the end of the day, and on the fourth, he has an accident at about that same time of day. He had an appointment with his doctor the next day. We collected responses to this scenario, discussing accountability, preventive measures and legal considerations.

As we aimed to focus on patient-generated and patient-reported data, we did not include other types of data where the patient does not intervene in data sharing after 
giving her initial consent (e.g., passive data such as EKG tracings from pace-makers, are collected automatically via a medical device, and are transmitted to an app or cloud).

\section{Results}

Apart from four physicians who took part in a focus group, we encountered the participants during individual interviews. Two patients, an informaticist, a legal representative, and two physicians (hospital and ambulatory care) were included. Two ambulatory care physicians, one hospitalist, and a hospital and ambulatory care physician took part in the focus group.

The care-providers all immediately recognized the potential benefits of including PGHD in the EMR to improve patient care, as they provide additional data points to help understand health issues or to adapt treatments (e.g., glucose or blood pressure). This is particularly the case for quantifiable measures such as glucose results or blood pressure measurements.

It was important for the clinicians that PGHD be easily distinguishable from clinician-collected data: dates are not sufficient to identify PGHD, as it can also be generated during hospital stays. For example, some individuals with diabetes continue checking their own blood glucose levels, even when they are hospitalized. The source of data is important because of the reliability that clinicians attribute to the results. Some blood pressure measurement devices (wrist devices, for example) that patients use may produce less reliable values than the hospital devices.

Healthcare providers were also interested in sending questionnaires to the patients, either to be completed at home or even in the waiting-room before a visit. These results can serve two purposes: quality assessment and improvement at an institutional level, and quality assessment and adaptation of treatment at the patient's individual level. For the first purpose, integration in the EMR chart is low priority, since it is the pooled results that are analyzed. For the second, integrating PGHD in the EMR is very important for patient care, and may be a key element for clinicians to adopt the use of PGHD.

Our scenarios raised several questions about shared PGHDs. In both situations, clinicians expressed concerns about the accountability for uploaded data. Physicians cannot always be checking if a patient uploads data in the EMR, nor do they have time to analyze all the data, even with the support of a care-provider team.

For the legal representative, all tests initiated by the healthcare provider must be followed up on, with the adequate action if needed. When these tests are conducted by the patient, the provider must provide the patient sufficient guidance to understand and react to the results if needed: responses can be to contact a healthcare provider, or selfmanagement measures (e.g., taking sugar for hypoglycemia). As soon as PGHD are shared by the patient with his provider, a clear understanding must be given to the patient about the expected action and accountability from the healthcare team. For example, a statement that answers are not seen by the provider outside of office hours would be particularly important for suicide issues. Another approach to decrease liability is to limit when the patient can upload data. In some cases, such as blood glucose results, data upload could occur prior to the next visit, and a window of 48 hours was suggested during the focus group.

Although this limited upload is beneficial to preserve provider liability, it could decrease the sharing of PGHD by patients. Interestingly, our patient participants 
interpreted this message positively, because it implied that providers would be looking at the data.

Several barriers can be identified when attempting to use PGHD to improve healthcare. First, patients need to be willing to share their data. Willingness to share data is affected by perceived benefits and expectations: one patient explained that when doctors do not look at a patient's glucose values during a visit, for example, patients will rapidly lose interest in testing their glucose levels, and will report them even less. This feedback loop can play an important in a patient's self-management. Although patients understand that some questionnaires may be needed for quality improvement at the institutional level, they expect individual responses to have an impact on their care, whenever possible.

Second, there may be interoperability issues, with data mainly accessible in proprietary software: although this in itself may not be a barrier for one health professional and one type of data for one patient, if each patient uses different apps to collect data, and patients differ in their choices of apps, health professionals do not want to $\log$ in to each of these to access a patients' PGHD. Beyond the time needed to log into the various apps, the scattering of the data in several sources makes it hard for the doctor to get an overview of a patient's health status. Finally, even if all the PGHD were collected in a single place for a provider to review, providers raised other concerns: they would still need to navigate back and forth between the EMR and the PGHD platform, and the amounts of data may be too vast for them to review appropriately. Therefore, PGHD should ideally be visualized in the places where similar data is collected by clinicians.

\section{Discussion}

The interviews and focus group point out some key elements in using PGHD in improving patient care. Beside the often cited interoperability and confidentiality issues, it is important to clarify the expected benefits and perceived risks for both the patient and provider users $[4,6,8]$. For patients, the feedback loop from the provider is a driver for continuing to self-test or to answer a questionnaire. Patients can accept a time-frame to upload data if it helps ensure that their data is analyzed. For example, this could be during the $48 \mathrm{~h}$ prior to the next planned visit: in fact, a reminder to upload PGHD could even be included in the visit reminder text messages that are growing in popularity.

Another feedback mechanism that could help motivate patients to fill out questionnaires and share PGHD would be to provide patients with visualisations of the results, both at an individual level (changes in monitored PGHD for example) as well as for the institutional level (i.e., how one individual's perspectives compare to the general population) [9].

For providers, the potential benefits of PGHD seemed obvious. One of the main concerns however was the required effort to access PGHD: integration of PGHD into the provider's electronic tool is essential, as it removes a barrier for clinicians to using PGHD. Ideally, PGHD should be visualized with other similar data in the EMR (i.e., glucose values together), but differentiated from the other data in the EMR. Filters can be useful for clinicians to view the data with or without PGHD, for example.

A major concern about PGHD for clinicians is data overload, and subsequent accountability [10]. Besides setting a timeframe for upload, certain data may require developing analytical tools [11]: providers do not wish to be alerted to every low blood 
glucose level, but may want to rapidly see the frequency of occurrence of this type of event, as well as if there is a pattern for recurrence. In the case of our scenario, the ideal system could have detected the pattern by the third occurrence, and notified the provider. Decreasing the morning insulin dose could have prevented the accident. In terms of liability, clinicians are accountable for dealing with abnormal results, and therefore need to be explicit about when they will review the uploaded data [12]. Patient expectations need to be aligned to avoid incidents(e.g., suicidal thoughts). Therefore, integrating PGHD will need to consider how to develop tools to screen PGHD to detect both abnormal responses, as well as abnormal patterns, for both structured and unstructured data.

\section{Conclusion}

We discussed the possibilities and challenges of using PGHD in patient care with major stakeholders through two scenarios with PGHD. Besides the importance of fully integrating PGHD in the EMR to facilitate its use for care, clinicians underlined the importance of comparing and distinguishing PGHD from other data. In terms of liability, it is essential to define PGHD upload conditions and analyses by the care-providers. Furthermore, future tools need to be developed to help screen for PGHD anomalies, including patterns of abnormality, in both structured and unstructured data, to improve the safety of patient care. The patients' positive appreciation of sharing conditions and clarified expectations in our study needs to be re-assessed in a larger population.

\section{References}

[1] Shapiro M, Johnston D, Wald J, Mon D. Patient-Generated Health Data - White Paper. Prep Off Policy Planning, Off Natl Coord Heal Inf Technol Res Triangle Park NC RTI Int. 2012;(April):35.

[2] Cohen DJ, Keller SR, Hayes GR, Dorr DA, Ash JS, Sittig DF. Integrating Patient-Generated Health Data Into Clinical Care Settings or Clinical Decision-Making: Lessons Learned From Project HealthDesign. JMIR Hum Factors. 2016;3(2):e26.

[3] Nittas V, Lun P, Ehrler F, Puhan MA, Mütsch M. Electronic Patient-Generated Health Data to Facilitate Disease Prevention and Health Promotion: Scoping Review. J Med Internet Res. 2019;21(10).

[4] Lavallee DC, Lee JR, Austin E, Bloch R, Lawrence SO, McCall D, et al. mHealth and patient generated health data: stakeholder perspectives on opportunities and barriers for transforming healthcare. mHealth. 2020;6(January):8-8.

[5] Demiris G, Iribarren SJ, Sward K, Lee S, Yang R. Patient generated health data use in clinical practice: A systematic review. Nurs Outlook. 2019;67(4):311-30.

[6] Genes N, Violante S, Cetrangol C, Rogers L, Schadt EE, Chan Y-FY. From smartphone to EHR: a case report on integrating patient-generated health data. npj Digit Med. 2018;1(1).

[7] Wilson V. Focus Groups: ? Br Educ Res J. 1997 Apr;23(2):209-24.

[8] Papautsky EL, Panganiban AR. Implications of Trust on Patient Generated Health Data. Proc Hum Factors Ergon Soc Annu Meet. 2018 Sep 27;62(1):550-550.

[9] DiClemente CC, Marinilli AS, Singh M, Bellino LE. The Role of Feedback in the Process of Health Behavior Changetle. Am J Health Behav. 2001;25(3):217-27.

[10] Chung AE, Basch EM. Potential and challenges of patient-generated health data for high-quality cancer care. J Oncol Pract. 2015;11(3):195-7.

[11] Sutton RT, Pincock D, Baumgart DC, Sadowski DC, Fedorak RN, Kroeker KI. An overview of clinical decision support systems: benefits, risks, and strategies for success. npj Digit Med. 2020;3(1):1-10.

[12] Pine KH, Bossen C, Chen Y, Ellingsen G, Grisot M, Mazmanian M, et al. Data Work in Healthcare. In: Companion of the 2018 ACM Conference on Computer Supported Cooperative Work and Social Computing. New York, NY, USA: ACM; 2018. p. 433-9. 